\title{
Novel approaches for the surgical treatment of atrial fibrillation:Time for a guideline revision?
}

\author{
This article was published in the following Dove Press journal: \\ Vascular Health and Risk Management \\ 4 June 2010 \\ Number of times this article has been viewed
}

\section{Carlo Nicola De Cecco ${ }^{1,2}$ \\ Vitaliano Buffa' \\ Vincenzo David² \\ Stefano Fedeli ${ }^{1}$ \\ 'Department of Cardiovascular Radiology, San Camillo-Forlanini Hospital, ${ }^{2}$ Department of Radiological Sciences, University of Rome, St Andrea Hospital, Rome, Italy}

Correspondence: Carlo Nicola De Cecco Department of Cardiovascular Radiology, San Camillo-Forlanini Hospital,Via Portuense 332, 00149 Rome, Italy

Tel +39335 5259926

Fax +39064 4260834

Email carlodececco@gmail.com

\begin{abstract}
Atrial fibrillation is a major health problem in Western countries, and is associated with considerable morbidity and resource consumption. Safe and reliable surgical techniques for the termination of this arrhythmia have been developed since the time of the original Cox "maze I" procedure. Novel equipment based on radiofrequency and microwave technologies can be employed to create transmural atrial lesions, even in the context of minimally invasive surgery to the atrioventricular valves via right minithoracotomy. The aim of this paper is to review the recent literature on this approach, and the clinical results in terms of arrhythmia termination and postoperative morbidity. With the aim to substantiate the practice of a simple, yet reliable, surgical ablation during minimally invasive heart valve surgery, we discuss the results of different patterns of atrial lesions having different degrees of surgical complexity. Finally, minimally invasive epicardial ablation for lone atrial fibrillation represents an emerging surgical indication. The results of state-of-the-art transcatheter ablation represent now its benchmark of comparison.
\end{abstract}

Keywords: atrial fibrillation, surgery, minimally invasive, outcomes

\section{Introduction}

Atrial fibrillation (AF) is the most common cardiac rhythm disturbance in adults, and is potentially associated with important cardiovascular morbidity and mortality, mainly due to thromboembolism. ${ }^{1}$ The association with mitral valvular disease is well known. The rate of failure of medical rhythm control therapy may be as high as $85 \%$ at two years. ${ }^{2}$

Despite adequate correction of valvular disease, AF is expected to persist postoperatively in those patients undergoing an operation on the mitral valve, and with persistent AF or paroxysmal AF lasting more than one year. ${ }^{3}$ This observation, coupled with the encouraging results of the Cox maze procedure as a treatment to restore sinus rhythm in patients with structural heart disease, ${ }^{4,5}$ has justified the practice of performing arrhythmia surgery in these individuals. This practice has been recently supported by a controlled randomized trial ${ }^{6}$ which highlighted the superiority of the radiofrequency maze ablation procedure in restoring sinus rhythm versus postoperative rhythm control treatment.

In the current era, minimally invasive strategies are becoming the standard of care for surgery to the atrioventricular valves. While these procedures are proven to be reproducible and their number is increasing, the research has focused on the development of equipment designed for surgical AF ablation via minimally invasive access. The aim 
of this paper is to review the current literature systematically regarding the feasibility, reliability, and surgical results of minimally invasive surgical techniques for the treatment of AF versus those obtained via the conventional surgical route, with particular attention to the results of different ablation line patterns.

\section{Methods}

A literature search was conducted for the period December 2009-January 2010 using the Medline-National Library of Medicine online database. The search was limited to English language prospective or retrospective studies of surgical epicardial AF ablation via an access different to that of conventional median sternotomy, with or without concomitant surgery to the atrioventricular valve(s). The terms "atrial fibrillation", "ablation", "surgical treatment", "minimally invasive", and "thoracotomy" were entered in the search heading. Further inclusion criteria were presentation of both early postoperative results and a clinical follow-up lasting at least 12 months for arrhythmia recurrence, reporting of data on late complications (including stroke, heart block, and pacemaker implantation) and on mortality. Study quality was evaluated in a blinded fashion by two investigators. Duplicate studies published on the same patient population were excluded. Divergent judgments were resolved by consensus of the investigators. For the purposes of the study, full median sternotomy was defined as the "conventional surgical route" for AF ablation, irrespective of the technique, energy source, and lesion pattern adopted to perform the ablation, with or without concomitant surgery for structural heart disease. The "minimally invasive approach" was defined as any approach entailing surgical incision other than median sternotomy (ie, thoracotomy, minithoracotomy, either with direct vision or thoracoscopy), with or without surgery for structural heart disease (Figures 1 and 2). Finally, in the present paper, no differentiation in data extraction has been made between the slightly different types of minimally invasive technique used to approach the heart (ie, fully thoracoscopic versus non-fully thoracoscopic systems).

Subsequently, sample size calculation for a prospective study comparing the follow-up results of transcatheter versus epicardial ablation of lone AF was performed. As a point of
A

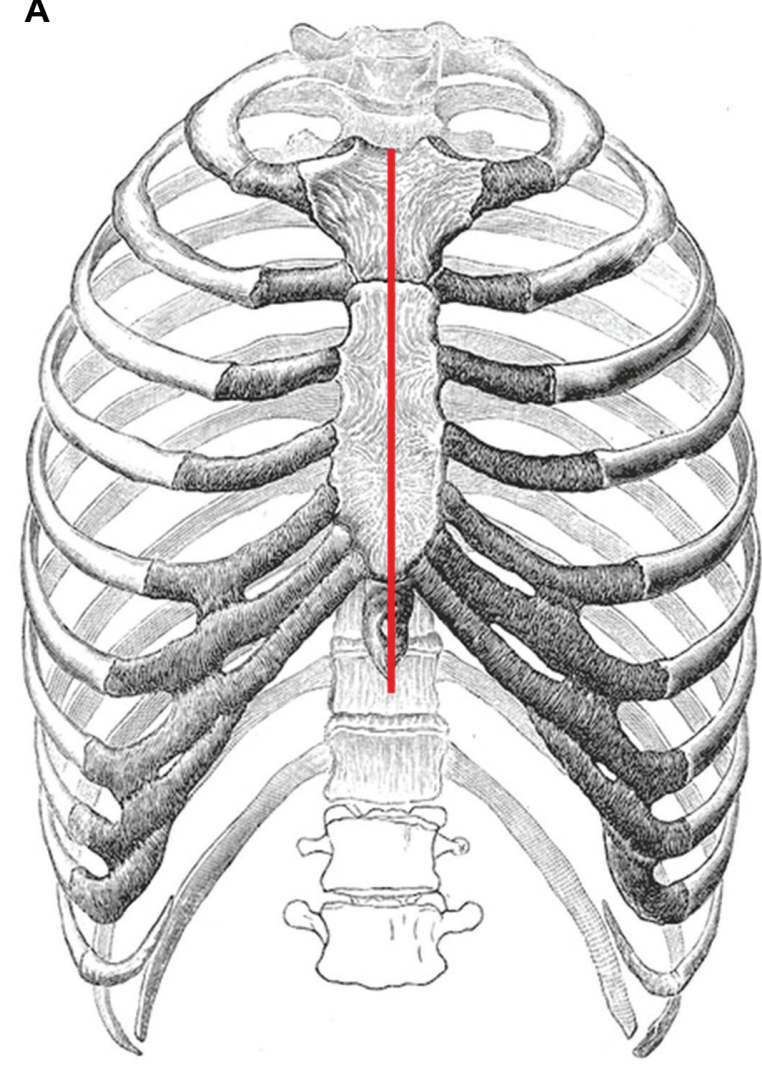

B

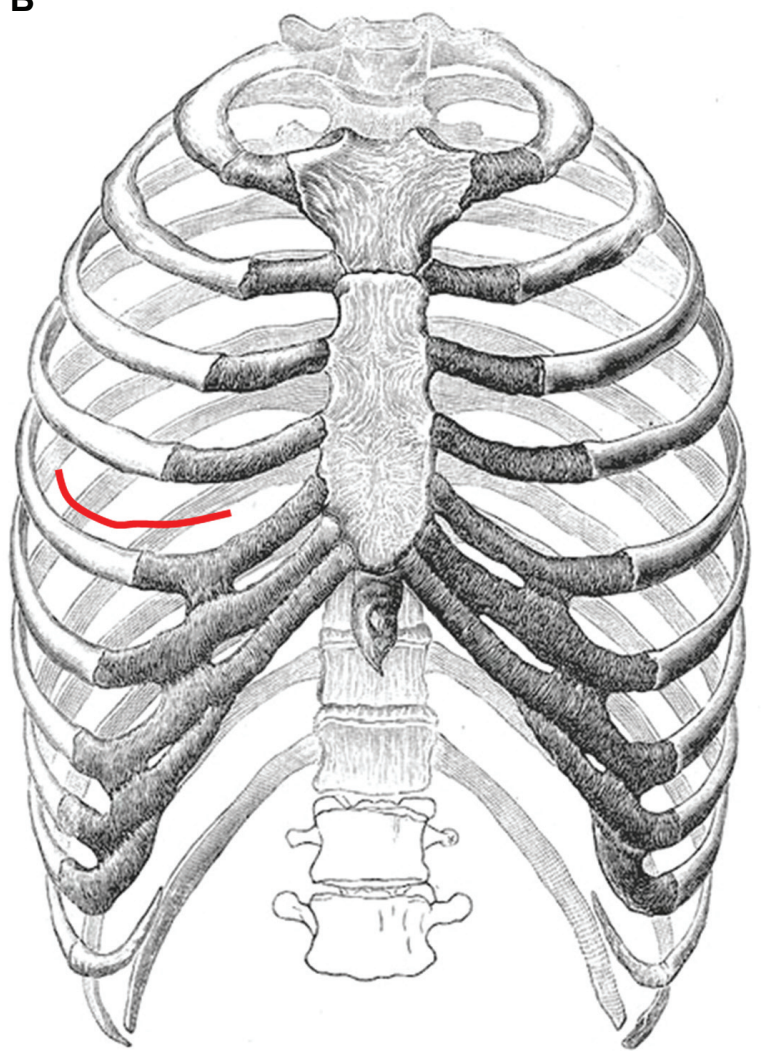

Figure I Schematic drawing of surgical incisions. A) Full median sternotomy entails complete division of the sternal bone in the midline in order to expose the heart and the great vessels. B) The minimally invasive approaches for surgery to the atrioventricular valve(s) and AF ablation entail a right minithoracotomy, most frequently in the fourth intercostal space, the length of which ranges from $3 \mathrm{~cm}$ to $8-9 \mathrm{~cm}$, depending on the technique.

Abbreviation: AF, atrial fibrillation. 


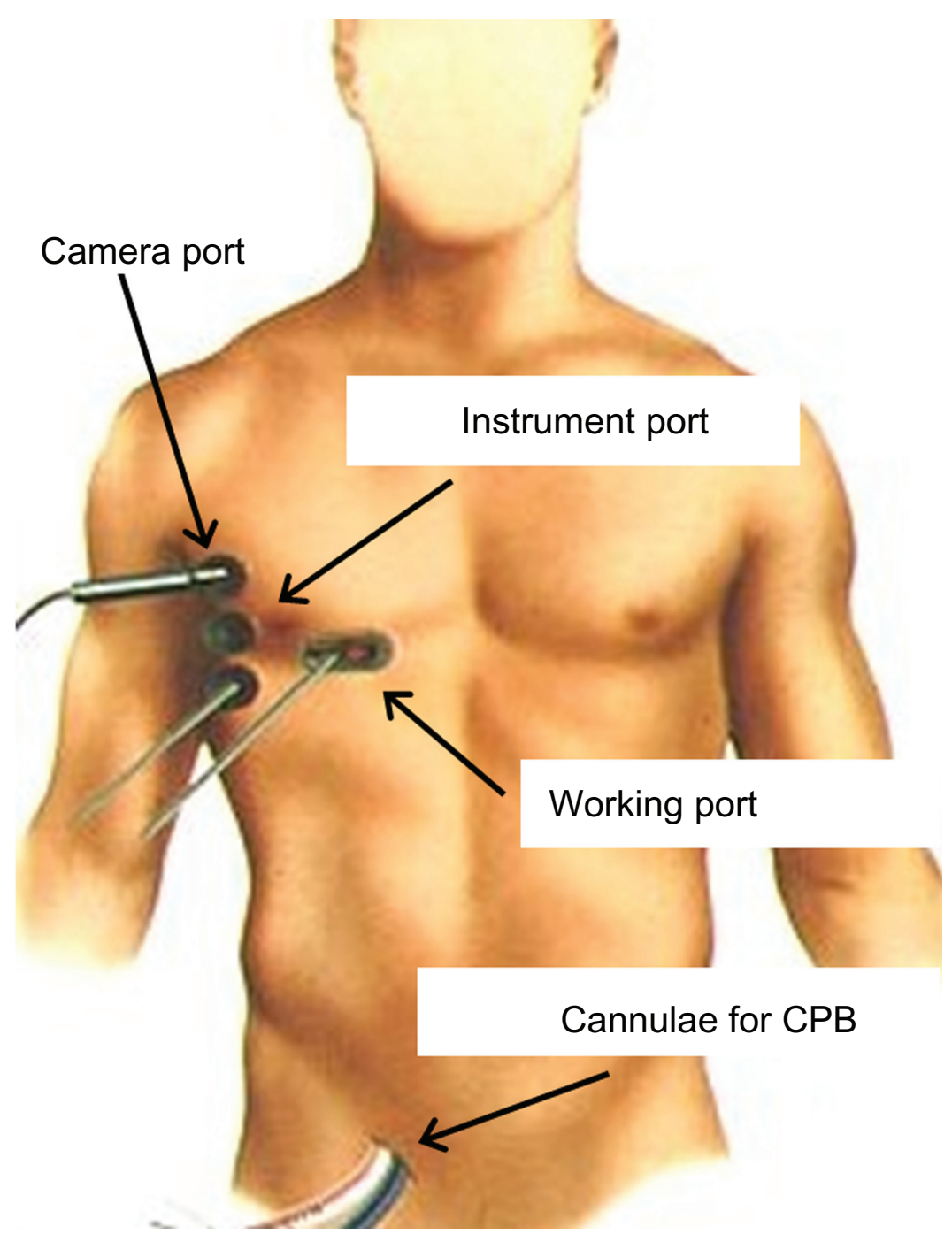

Figure 2 Setup of the port access system. Cardiopulmonary bypass is established by cannulation of the common femoral vessels (both for venous drainage from the inferior vena cava and arterial inflow in the common femoral artery). A second venous drainage cannula is placed in the superior vena cava through the right internal jugular vein. A right minithoracotomy in the inframammary groove is used to lead prosthetic material into the chest. A camera port is used to provide full videoscopic assistance during the procedure, and three additional instrument ports are used. An expandable balloon inserted through the femoral artery is used to clamp the ascending aorta from the inside (aortic endoclamp), and to deliver cardioplegia to protect the myocardium during the arrest period.

reference, we used follow-up results of patients subjected to transcatheter ablation and to combined cardiac surgery and AF ablation in our institution. The endpoints used in our analysis were rate of recurrent $\mathrm{AF}$ at hospital discharge and rate of recurrent $\mathrm{AF}$ at the six-month follow-up. A 0.9 statistical power was applied, and the analysis was conducted using the PS Power and Sample Size calculation software ver. 3.0 for Windows.

\section{Epicardial ablation via full median sternotomy}

A pooled meta-analysis investigation published in 2005 has confirmed the usefulness of AF ablation during mitral surgery to maintain sinus rhythm and to reduce the risk of stroke during follow-up versus mitral surgery alone. The association between the maze procedure and increased risk of postoperative pacemaker requirement, as mentioned in several previous studies, was also confirmed. However, a proportion of these cases may theoretically represent unmasked pre-existing sinus node dysfunction; thus, pacemaker implantation may not be attributable to the surgical procedure itself in all individuals. Discrimination among these two patient categories needs to be researched further. In the same meta-analysis, an increased rate of postoperative revision for bleeding was associated with AF ablation. As such, bleeding in these patients is almost entirely attributable to the atrial incisions of the "cut-and-sew" technique. In fact, the incidence of revision for bleeding in the literature included in this meta-analysis is 
consistently lower whenever RF or cryoablation techniques were used. $^{7-15}$

Despite continuing methodologic improvements, the success rates with transcatheter endocardial ablation for AF may be less than optimal, despite employment of stateof-the-art technologies. It emerged from randomized trials that the coupling of pulmonary vein isolation with left atrial linear lesions through the mitral annulus and the left atrial appendage may improve the results. ${ }^{16}$ However, reported success rates approach $57 \%$ at one year and $53 \%$ at three years. A second ablation procedure or more complex and staged ablation patterns may improve the outcome. ${ }^{17}$ Such complex transcatheter ablation strategies may not be costeffective in a time of escalating medical costs. ${ }^{18}$ Conversely, epicardial left atrial ablation using current radiofrequency technology has a success rate of $85 \%$ at two years after one single procedure. ${ }^{19}$ Other papers have reported a $94 \%$ rhythm control rate at follow-up if additional lesions are produced within the right atrium. ${ }^{8}$ Nonetheless, a median sternotomy incision has been the price of performing a maze procedure for a long time, with its ensuing morbidity, hospitalization, and prolonged postprocedural recovery. These factors have rendered epicardial ablation unattractive to both patients and their referring physicians, unless cardiac surgery is indicated for other reasons.

\section{Emerging techniques: review of clinical results}

The 2006 American College of Cardiology/American Heart Association/European Society for Cardiology guidelines for the management of patients with AF have cited minimally invasive surgical techniques for atrial fibrillation ablation as a work-in-progress at the research level. ${ }^{20}$ However, the minimally invasive approach, with its lesser ensuing morbidity and increased tolerability for patients, was underscored to have the potential to reform the algorithms for treatment of AF, provided that it is as reliable as the gold standard techniques.

\section{Choice of the lesion set: implications for minimally invasive approaches}

Both a biatrial lesion set and an ablation procedure confined to the left atrium can be performed via the minimally invasive approach. However, a reliable yet fast and safe procedure for AF ablation is even more important in this context. Minimally invasive surgery is associated with a higher level of technical complexity, prolonged operative times, ${ }^{21}$ and on occasion, a more difficult exposure. Extra cardiopulmonary bypass time is required to perform a biatrial lesion pattern, with additional right atriotomy when surgery to the mitral valve alone is scheduled. Although a biatrial classic maze is technically achievable by a minimally invasive route, ${ }^{8}$ the industry has produced several disposable devices designed for minimally invasive epicardial ablation. All these devices are based on a left atrial, beating heart ablation concept. Such characteristics can be particularly advantageous in the minimally invasive setting. It has been ascertained that the pathophysiologic substrate of AF can vary from simpler forms (arrhythmogenic foci around the pulmonary veins) to more complex electrophysiologic patterns involving both the atria. ${ }^{22,23}$ Unfortunately almost all mapping data available in the literature have been obtained from nonsurgical patients with lone AF, and in such patient populations the AF displays a biatrial substrate in the majority of cases. ${ }^{24}$ Furthermore, patients coming for surgery to the atrioventricular valve(s) may represent a distinct subgroup displaying predominantly the same pathophysiologic mechanism for AF.

The established Cox maze procedure entails a complex set of atrial lesions involving both the atria (Figure 3). Ablation procedures confined to the left atrium consist of isolation of the pulmonary veins either using the "en bloc" technique (a single ablation line encircling the four vein orifices altogether) or by isolating separately the right and left pulmonary veins cuffs. A "mitral line" usually completes this procedure by connecting the pulmonary vein line to the mitral annulus, and can be performed either endocardially or epicardially. The biatrial procedures may add to this set a cavocaval ablation line, and other lesions directed through the coronary sinus, tricuspid orifice, and interatrial septum on the right atrial side. Amputation or isolation of the right/left atrial appendages may complete the procedure. A number of modifications have been proposed to this lesion set, ${ }^{25}$ while different energy sources have almost entirely replaced the original "cut-and-sew" technique (radiofrequency, cryoablation, ultrasound, and microwave energy). These are less technically demanding and their results, in terms of postoperative sinus rhythm conversion, have been reported in a systematic review to be not significantly different from those claimed after the "cut-and-sew" approach. ${ }^{26}$ A clear relationship between the maintenance of postoperative sinus rhythm and the type of lesion pattern (biatrial versus left atrial) could not be established in the same meta-regression analysis. On the contrary, lesion transmurality appears to be an important determinant of therapeutic success. While ablation within the left atrium 

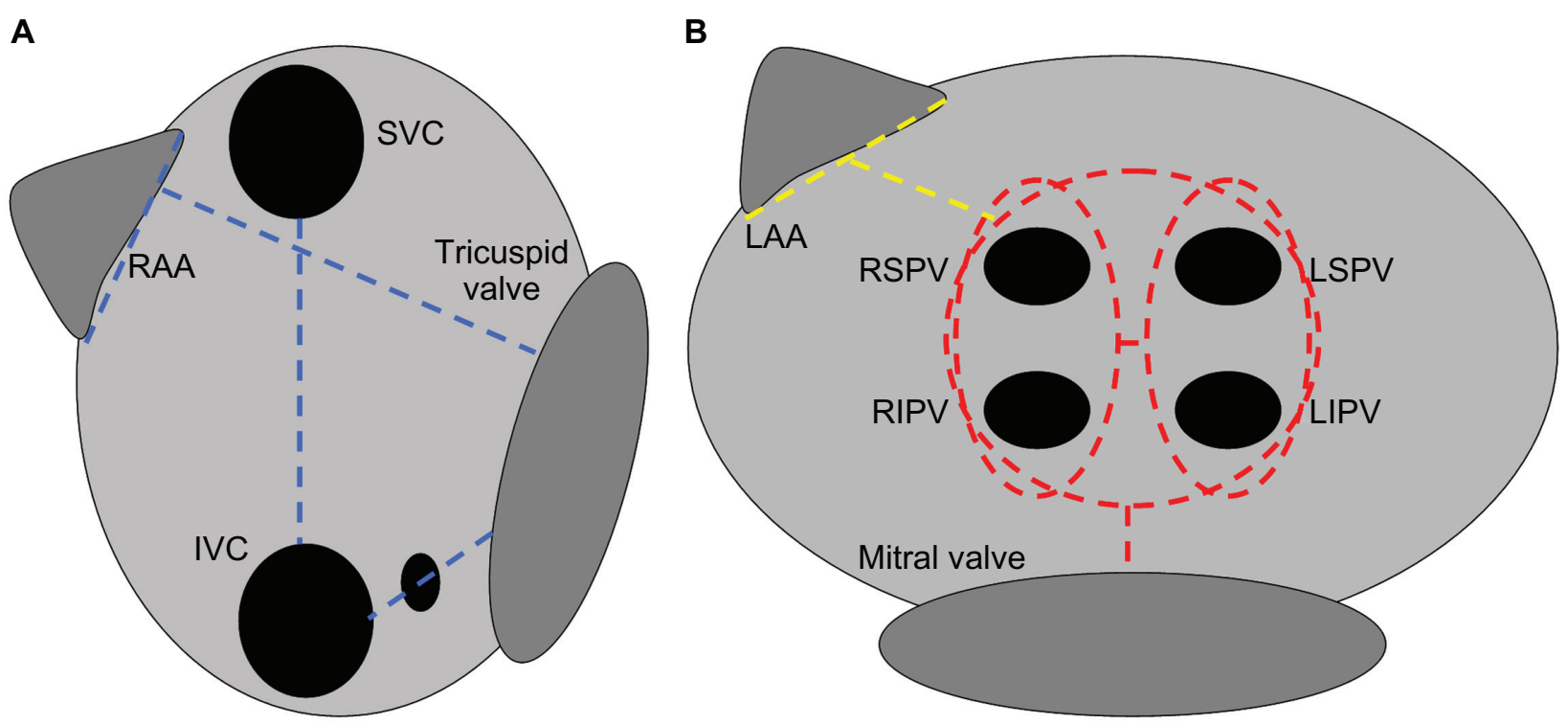

Figure 3 A) Left atrial lesions. Red interrupted line: Circular "box lesion" around the pulmonary vein orifices (or isolation of the left and right pulmonary cuffs independently) plus a lesion towards the mitral annulus ("mitral line"). Yellow interrupted line: Supplementary left atrial lesions, isolation of the left atrial appendage plus connecting line. B) Right atrial lesions. Cavocaval line, cavotricuspid line, isolation of the right atrial appendage plus connecting line towards the tricuspid annulus (the latter line is on the lateral right atrial wall).

Abbreviations: RSPV, right superior pulmonary vein; RIPV, right inferior pulmonary vein; LSPV, left superior pulmonary vein; LIPV, left inferior pulmonary vein; RAA, right atrial appendage; LAA, left atrial appendage; SVC, superior vena cava; IVC, inferior vena cava.

is essential for any surgical treatment of AF, the creation of supplementary right-sided lesions may increase the risk of pacemaker requirement. ${ }^{20,27}$

The evidence collected so far for the reliability of biatrial surgical ablation procedures versus those confined to the left atrium is conflicting. In a meta-analysis, the biatrial procedures appeared to confer superior results in terms of maintenance of sinus rhythm over time. ${ }^{28}$ However, this meta-analysis included studies published in the 1995-2005 period, and is limited by the inclusion of several retrospective trials. Evidence has been collected showing that isolation of the pulmonary veins coupled with creation of a "mitral line" is actually enough to interrupt the macro-reentrant circuit in cases of AF associated with mitral valvular disease. ${ }^{29}$ Meanwhile, the lesions within the right atrium, septum, and base of the left atrial appendage may not confer a significant benefit in terms of major clinical outcomes (maintenance of sinus rhythm and thromboembolic events).$^{30}$ Controlled randomized trials addressing these issues have been recently published. The largest of these studies randomized 299 patients scheduled for cardiac surgery via median sternotomy to receive radiofrequency AF ablation with either a left atrial technique (pulmonary vein isolation plus connecting lines to the mitral annulus and atrial roof) or a biatrial technique (adding cavocaval, cavotricuspid isthmus, and lateral free wall lesions to the previous set). ${ }^{19}$ The percentage of patients in sinus rhythm at the end of a mean 28-month follow-up was comparable among the study groups. A markedly increased left atrial diameter $(>80 \mathrm{~mm})$ was the sole significant predictor of late AF recurrence. In another randomized investigation, the early and late results of pulmonary vein isolation plus creation of a "mitral line" were similar to that after the Cox maze III procedure in the context of mitral surgery ${ }^{31}$ The clinical outcomes were superior in both groups versus the control patients, who received no ablation at all. Two other controlled trials yielded similar conclusions. ${ }^{7,32}$ The comparability of outcomes for left atrial and biatrial procedures apparently is not offset by the type of left atrial lesion set, namely whether the isolation of the left atrial appendage and creation of a "mitral line" are added to pulmonary vein isolation..$^{33}$ The latter randomized study was conducted in a homogeneous group of patients undergoing surgery to the atrioventricular valve(s) and cryoablation. The fairly low observed rates of freedom from recurrent AF (57\%-67\%) have been attributed to the practice of not administering antiarrhythmic drugs in the perioperative period during this study. This observation indirectly confirms the usefulness of pharmacologic therapy to optimize the results of surgical ablation. Although no relevant trials have been conducted to date, there is no suggestion in the literature of different outcomes depending on the type of pulmonary vein isolation (en bloc versus separate left and right cuff ablation). Finally, 
it is noticeable that the rate of freedom from recurrent AF at follow-up after intraoperative radiofrequency ablation for permanent AF has progressively improved over time. In the early 2000 s a freedom rate approaching $70 \%$ was considered to be an adequate result, ${ }^{9,34}$ while freedom rates are now approximately $90 \%{ }^{5,10,9}$

Thus, the conclusions of the meta-analysis by Barnett et al should be critically revised on the basis of this evidence, and probably reperformed including randomized controlled trials alone. A summary of the recent evidence from randomized studies is reported in Table 1. These data are a theoretical basis for the development of disposable instruments providing epicardial, offpump AF ablation by circular lesion around the pulmonary vein orifices. The radiofrequency or highintensity focused ultrasound technologies are invariably used for these purposes. Tip-cooling and vacuum-assistance are used in some cases, in order to facilitate the adherence of the ablation probe to the beating cardiac tissue and the creation of a transmural lesion. Initial reports exist for the results of these devices in the context of median sternotomy. The Epicor Cardiac Ablation System (St Jude Medical, St Paul, $\mathrm{MN}$ ) enables offpump creation of a circular box lesion around the pulmonary veins and the mitral line using two different devices, with high-intensity ultrasound energy. After setting up the system, the surgeon can proceed with preparation for cardiopulmonary bypass as required, thus saving both operative and pump time. This device can be potentially used via a minimally invasive route, and in the only published experience, a 77\% rate of freedom from recurrent arrhythmia at follow-up was reported. ${ }^{35}$ However, few patients $(n=14)$ were included in this research and larger data sets are required. Initial clinical data are available also for devices designed specifically for use in minimally invasive settings, eg, the

Table I Summary of randomized controlled trials comparing the results of biatrial versus left atrial ablation procedures published in the 2005-2009 period $^{*}$

\begin{tabular}{lllll}
\hline Study & $\begin{array}{l}\text { Sample } \\
\text { size (n) }\end{array}$ & $\begin{array}{l}\text { Left atrial: SR } \\
\text { at follow-up }\end{array}$ & $\begin{array}{l}\text { Biatrial: SR } \\
\text { at follow-up }\end{array}$ & $\boldsymbol{P}^{*}$ \\
\hline Wang et $\mathrm{al}^{19}$ & 299 & $85 \%$ & $84 \%$ & 0.87 \\
${\text { Albrecht et } \mathrm{al}^{31}}^{31}$ & 60 & $90 \%^{\S}$ & $85 \%$ & 0.21 \\
${\text { Srivastava et } \mathrm{al}^{33}}$ & 160 & $67.5 \%$ and $57.5 \%^{\dagger}$ & $62.5 \%$ & $\mathrm{NS}$ \\
\hline
\end{tabular}

"Probability value for intergroup comparison of primary outcome variable (maintenance of SR). In this study patients were randomized to four study groups, ie, biatrial ablation versus pulmonary vein isolation alone $(67.5 \%$ freedom from $\mathrm{AF}$ at follow-up) versus pulmonary vein isolation plus other left atrial ablation lines (57.5\%

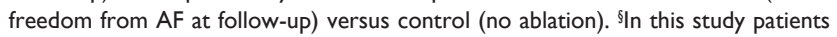
were randomized to three study groups, ie, pulmonary vein isolation and ablation ( $90 \%$ freedom from AF at follow-up) versus biatrial maze ( $85 \%$ freedom from $\mathrm{AF}$ at follow-up) versus controls (no ablation).

Abbreviations: SR, sinus rhythm; NS, not significant; AF, atrial fibrillation.
Cobra Adhere XL System (Estech Inc., San Ramon, CA) which uses radiofrequency energy, and the Flex 10 Ablation System (Guidant Inc., Indianapolis, IN) which uses microwave energy. ${ }^{36,37}$ Both devices rely on the creation of a box lesion around the pulmonary veins; however, the reported rates of freedom from $\mathrm{AF}$ at follow-up were markedly different for these devices ( $92 \%$ versus $42 \%$, respectively). This discrepancy may be partially accounted for by population bias because the patients enrolled in the first study received minimally invasive surgery for mitral disease and concomitant AF ablation, whereas the patients in the second study had lone AF. This should prompt caution when considering the extension of the indication for surgical epicardial ablation to individuals with lone AF. However, the authors did comment that their disappointing results could be ascribed to ineffective creation of complete lesions, given that the microwave probe was not vacuum-assisted. ${ }^{37}$

\section{Advantages versus conventional surgical approach}

Full sternal splitting leads to alterations of the respiratory mechanics, thus impacting the postoperative pulmonary recovery, particularly in patients with chronic lung disease. Median sternotomy is a moderately painful incision and is prone to complications, which may range from serous discharge to sternal instability and life-threatening mediastinitis. Surgery to the mitral valve via a minimally invasive approach is a reproducible operation, either via minithoracotomy, peripheral cannulation, endoaortic clamping, and total videoscopic assistance (Port-Access system) ${ }^{38,39}$ or via minithoracotomy, peripheral cannulation, direct vision, and external aortic clamping. ${ }^{40} \mathrm{~A}$ recent propensity-matched investigation in a total of 715 patients showed that the minimally invasive strategy for cardiac surgery is associated with a shorter mechanical ventilation time, decreased postoperative pain, and improved patient satisfaction, although at the price of longer cardiopulmonary bypass and aortic clamping times. ${ }^{21}$ Concomitant $\mathrm{AF}$ ablation is feasible during minimally invasive surgery to the atrioventricular valve(s), with a reported $28 \%$ rate of arrhythmia recurrence at follow-up after port-access mitral repair/replacement plus radiofrequency isolation of the pulmonary veins. ${ }^{41} \mathrm{~A}$ unipolar radiofrequency instrument was used endocardially during cardiopulmonary bypass in that series. In a randomized study setting, a 93.6\% freedom from late AF was reported in patients receiving onpump endocardial radiofrequency ablation during minimally invasive valvular surgery. ${ }^{8}$ In the majority of the cases included in that study a left atrial 
ablation procedure was performed, and the lesions were extended to the right side only in those cases requiring a procedure on the tricuspid valve or the interatrial septum. Unfortunately the authors did not report differential results of patients receiving the biatrial versus the left atrial procedure. However, this is predominantly a left-sided ablation series and the outstanding clinical results reported should be essentially considered as the expression of this strategy. The left atrial lesion sets included isolation of the pulmonary vein cuffs and left atrial appendage and creation of a "mitral line". In a direct comparison of cryomaze either through conventional sternotomy or with a minimally invasive system, comparable rates of follow-up arrhythmia recurrence $(2.2 \%$ versus $2.4 \%$ ) and comparable rates of major perioperative morbidity were observed. The rate of postoperative permanent pacemaker requirement was reduced, possibly due to enhanced videoscopic exposure of the tissue targets. ${ }^{25}$ The possibility of a considerable incidence of hemidiaphragm paralysis was also noticed, as a consequence of phrenic nerve trauma while entering the pericardium from the right pleural cavity.

In general terms, the minimally invasive ablation strategies have proved to be noninferior in their main clinical results to ablation via full sternotomy. A left atrial lesion set, coupled with adequate perioperative pharmacologic therapy, appears to be feasible via right minithoracotomy. The known advantages of minimally invasive valvular surgery (patient satisfaction, shorter ventilation, and perhaps reduced morbidity) are not offset by concomitant ablation. Additionally, AF ablation can be performed during minimally invasive procedures other than surgery on the atrioventricular valve(s), thus expanding its potential field of application.

\section{Future implications: surgical ablation of lone AF}

Unfortunately the currently available data are insufficient to perform a formal meta-analysis of the clinical results of radiofrequency AF ablation via the minimally invasive route versus conventional median sternotomy. Postoperative length of stay, morbidity, and rate of maintenance of sinus rhythm should be included among the endpoints of such a meta-analysis.

Although still inferential, it can be reasonably expected that patients undergoing epicardial ablation through a minimally invasive access would have benefits similar to those observed after minimally invasive mitral valve surgery versus surgery via the conventional approach. However, the presence of mitral valvular disease in these patients represents a major confounder. Comparing the gross rates of freedom from AF after surgical ablation versus after state-of-the-art transcatheter procedures could be inherently misleading, because different pathophysiologic mechanisms may subtend the arrhythmia in these circumstances. Trigger focuses within the pulmonary vein cuffs have been linked with paroxysmal AF, thus substantiating the excellent results of radiofrequency isolation of the pulmonary veins alone. ${ }^{42}$ In the setting of permanent AF, more advanced remodeling of the atrial wall may determine ubiquitous trigger foci. Nonetheless, macro-reentrant circuits around the pulmonary cuffs, particularly if atrial dilatation coexists, are believed to sustain the arrhythmia in this case. Pulmonary vein isolation and creation of a "mitral line" should then interrupt such a circuit. ${ }^{43}$ These issues can be properly addressed only by a direct comparison of nonvalvular AF ablation via the transcatheter versus the surgical technique, and by a deeper comprehension of the mechanisms triggering AF in different patient subgroups. For research purposes, pre- or intraoperative electrophysiologic study may be performed.

From the feasibility point of view, offpump, epicardial, minimally invasive ablation has a limited traumatic profile, reproducibility, and expected optimal clinical results. Cardiopulmonary bypass and ensuing multisystem morbidity, systemic inflammatory reaction, and prolonged hospitalization are avoided. These elements may prompt a more liberal policy to indicate minimally invasive epicardial ablation in patients with drug-refractory, lone AF. The performance of a randomized investigation versus transcatheter ablation is encouraged. Centers experienced in minimally invasive surgery to the atrioventricular valves may be the ideal candidates to lead this research. Early results for epicardial ablation of lone AF with high-intensity focused ultrasound technology via sternotomy have been encouraging. ${ }^{35}$ Further studies using minimally invasive equipments are ongoing.

Our sample size calculation analysis revealed that in a prospective study of transcatheter versus epicardial ablation of lone AF, each study group should include 125 subjects to have sufficient statistical power to detect a significant difference between groups in terms of rate of recurrent $\mathrm{AF}$ at hospital discharge and rate of recurrent $\mathrm{AF}$ at six-month follow-up.

\section{Conclusion}

Overall, the evidence indicates that a reliable AF ablation can be safely performed with a lesion set confined to the left atrium. To this end, disposable devices for epicardial offpump ablation in the minimally invasive setting have 
become available in recent years. With ongoing technological refinements it is reasonable to expect gold standard clinical results in terms of arrhythmia termination. Radiofrequency technology appears to be the most promising in this scenario. However, we can expect that the best results possible from minimally invasive ablation technologies can be achieved when the surgical indication is correctly posed. Hence, ongoing research is required.

As additional clinical data become available, these may suffice to support a revision of the published guidelines, given that the less traumatic profile of the minimally invasive techniques may allow for an extension of the surgical indications to patients with lone AF. There is a need for a randomized, controlled, multicenter trial comparing state-of-the-art transcatheter ablation versus minimally invasive epicardial, offpump ablation to treat lone AF. This would be the most reliable tool to ascertain on a clinical and cost-efficacy basis the relative place of these techniques in the management of this pandemic disease.

\section{Disclosure}

The authors report no conflicts of interest in this work.

\section{References}

1. Ezekowitz MD, Netrebko PI. Anticoagulation in management of atrial fibrillation. Curr Opin Cardiol. 2003;18:26-31.

2. Lundstrom T, Rydén L. Chronic atrial fibrillation. Long-term results of direct current conversion. Acta Med Scand. 1988;223:53-59.

3. Kawaguchi AT, Kosakai Y, Sasako Y, Eishi K, Nakano K, Kawashima Y. Risks and benefits of combined maze procedure for atrial fibrillation associated with organic heart disease. J Am Coll Cardiol. 1996; 28:991-993.

4. Cox JL, Boineau JP, Schuessler RB, Jaquiss RD, Lapass DG. Modifications of the maze procedure for atrial flutter and atrial fibrillation. I. Rationale and surgical results. J Thorac Cardiovasc Surg. 1995;110:473-484.

5. Cui YQ, Sun LB, Li Y, et al. Intraoperative modified Cox mini-maze for long-standing persistent atrial fibrillation. Ann Thorac Surg. 2008;85:1283-1289.

6. von Oppel U, Masani N, O'Callaghan P, Wheeler R, Dimitrakakis G, Sciffelers S. Mitral valve surgery plus concomitant atrial fibrillation ablation is superior to mitral valve surgery alone with an intensive rhythm control strategy. Eur J Cardiothorac Surg. 2009;35:641-650.

7. De Lima GG, Kalil RAK, Leiria TLL, et al. Randomized study of surgery for patients with permanent atrial fibrillation as a result of mitral valve disease. Ann Thorac Surg. 2004;77: 2089-2095.

8. Akpinar B, Guden M, Sagbas E, et al. Combined radiofrequency modified maze and mitral valve procedure through a port-access approach: Early and mid-term results. Eur J Cardiothorac Surg. 2003;24:223-230.

9. Deneke T, Khargi K, Grewe P, et al. Efficacy of an additional Maze procedure using cooled-tip radiofrequency ablation in patients with chronic atrial fibrillation and mitral valve disease. A randomized, prospective trial. Eur Heart J. 2002;23:558-566.
10. Jesserun ER, Van Hemel NM, Defauw JJ, et al. A randomized study of combining maze surgery for atrial fibrillation with mitral valve surgery. J Cardiovasc Surg. 2002;44: 9-18.

11. Bando K, Kobayashi J, Kosakai Y, et al. Impact of Cox maze procedure on outcome in patients with atrial fibrillation and mitral valve disease. J Thorac Cardiovasc Surg. 2002;124:575-583.

12. Handa N, Schaff HV, Morris JJ, Anderson BJ, Kopecky SL, EnriquezSarano M. Outcome of valve repair and Cox maze procedure for mitral regurgitation and associated atrial fibrillation. JThorac Cardiovasc Surg. 1999;118:628-635.

13. Chen MC, Chang JP, Guo GB, Chang HW. Atrial size reduction as a predictor of the success of radiofrequency maze procedure for chronic atrial fibrillation in patients undergoing concomitant valvular surgery. J Cardiovasc Electrophysiol. 2001;12:867-874.

14. Raanani E, Albage A, David TE, Yau TM, Armstrong S. The efficacy of the Cox/maze procedure combined with mitral valve surgery: A matched control study. Eur J Cardiothorac Surg. 2001;19:438-442.

15. Jatene MB, Marcial MB, Tarasoutchi F, Cardoso RA, Pomerantzeff P, Jatene AD. Influence of the maze procedure on the treatment of rheumatic atrial fibrillation - evaluation of rhythm control and clinical outcome in a comparative study. Eur J Cardiothorac Surg. 2000;17: $117-124$.

16. Gaita F, Caponi D, Scaglione M, et al. Long-term clinical results of two different ablation strategies in patients with paroxysmal and persistent atrial fibrillation. Circ Arrhythm Electrophysiol. 2008;14:269-275.

17. Di Biase L, Elayi CS, Fahmi TS, et al. Atrial fibrillation ablation strategies for paroxysmal patients: Randomized comparison between different techniques. Circ Arrhythm Electrophysiol. 2009;2:113-119.

18. Khaykin Y, Wang X, Natale A, et al. Cost comparison of ablation versus antiarrhythmic drugs as first-line therapy for atrial fibrillation: An economic evaluation of the RAAFT pilot study. J Cardiovasc Electrophysiol. 2009;20:7-12.

19. Wang J, Meng X, Li H, Cui Y, Han J, Xu C. Prospective randomized comparison of left atrial and biatrial radiofrequency ablation in the treatment of atrial fibrillation. Eur J Cardio-Thorac Surg. 2009;35:116-122.

20. Writing Committee Members. ACC/AHA/ESC 2006 guidelines for the management of patients with atrial fibrillation. Europace. 2006;8:651-745.

21. Suri R, Schaff HV, Meyer S, Hargrove WC. Thoracoscopic versus open mitral valve repair: A propensity score analysis of early outcomes. Ann Thorac Surg. 2009;88:1185-1190.

22. Schwartzman D, Bazaz R, Nosbisch J. Common left pulmonary vein: A consistent source of arrhythmogenic atrial ectopy. J Cardiovasc Electrophysiol. 2004;15:560-566.

23. Franz MR, Karasik PL, Li C, Moubarak J, Cjavez M. Electrical remodeling of the human atrium: Similar effects in patients with chronic atrial fibrillation and atrial flutter. J Am Coll Cardiol. 1997;30: $1785-1792$.

24. Rostock T, Steven D, Hoffmann B, et al. Chronic atrial fibrillation is a biatrial arrhythmia: Data from catheter ablation of chronic atrial fibrillation aiming for arrhythmia termination using a sequential ablation approach. Circ Arrhythm Electrophysiol. 2008;1:344-353.

25. Ad N, Cox JL. The maze procedure for the treatment of atrial fibrillation: A minimally invasive approach. $J$ Card Surg. 2004;19:196-200.

26. Khargi K, Hutten BA, Lemke B, Deneke T. Surgical treatment of atrial fibrillation: A systematic review. Eur J Cardiothorac Surg. 2005;25:258-265.

27. Gillinov AM, McCarthy PM. Advances in the surgical treatment of atrial fibrillation. Cardiol Clin. 2004;22:147-157.

28. Barnett S, Ad N. Surgical ablation as treatment for the elimination of atrial fibrillation: A meta-analysis. J Thorac Cardiovasc Surg. 2006;131:1029-1035.

29. Cox JL. Surgical treatment of atrial fibrillation: A review. Europace. 2003;5:S20-S29. 
30. Ninet J, Roques X, Seitelberger R, et al. Surgical ablation of atrial fibrillation with off-pump, epicardial, high-intensity focused ultrasound: Results of a multicenter trial. J Thorac Cardiovasc Surg. 2005;130:803-809.

31. Albrecht A, Kalil R, Schuch L, Abrahao R, et al. Randomized study of surgical isolation of the pulmonary veins for correction of permanent atrial fibrillation associated with mitral valve disease. J Thorac Cardiovasc Surg. 2009;138:454-459.

32. Abreu Filho C, Lisboa L, Dallan L, et al. Effectiveness of the maze procedure using cooled-tip radiofrequency ablation in patients with permanent atrial fibrillation and rheumatic mitral valve disease. Circulation. 2005;112:I20-I25.

33. Srivastava V, Kumar S, Javali S, et al. Efficacy of three different ablative procedures to treat atrial fibrillation in patients with valvular heart disease: A randomised trial. Heart Lung Circ. 2008;17:232-240.

34. Wellens F, Casselman F, Geelen P, et al. Combined atrial fibrillation and mitral valve surgery using radiofrequency technology. Semin Thorac Cardiovasc Surg. 2002;14:219-225.

35. Mitnovetski S, Almeida AA, Goldstein J, Pick AW, Smith JA. Epicardial high-intensity focused ultrasound cardiac ablation for surgical treatment of atrial fibrillation. Heart Lung Circ. 2009;18:28-31.

36. Bevilacqua S, Gasbarri T, Cerillo AG, et al. A new vacuum-assisted probe for minimally-invasive radiofrequency ablation. Ann Thorac Surg. 2009:1317-1321.
37. Pruitt JC, Lazzara RR, Ebra G. Minimally invasive surgical ablation of atrial fibrillation: The thoracoscopic box lesion approach. J Interv Card Electrophysiol. 2007;20:83-87.

38. Casselman F, Van Slycke S, Dom H, Lambrechts D, Vermeulen Y, Vanermen U. Endoscopic mitral valve repair: Feasible, reproducible, and durable. J Thorac Cardiovasc Surg. 2003;125:273-282.

39. Galloway A, Shemin R, Glower D, et al. First report of the port access international registry. Ann Thorac Surg. 1999;67:51-58.

40. Karimov JH, Bevilacqua S, Solinas M, Glauber M. Triple heart valve surgery through a right antero-lateral minithoracotomy. Interact Cardiovasc Thorac Surg. 2009;9:360-362.

41. Jeanmart H, Casselman F, Beelen R, et al. Modified maze during endoscopic mitral valve surgery: The OLV Clinic experience. Ann Thorac Surg. 2006;82:1765-1769.

42. Benussi S, Nascimbene S, Agricola E, et al. Surgical ablation of atrial fibrillation using the epicardial radiofrequency approach: Mid-term results and risk analysis. Ann Thorac Surg. 2002;74:1050-1056.

43. Mohr FW, Fabricius AM, Falk V, et al. Curative treatment of atrial fibrillation with intraoperative radiofrequency ablation: Short-term and midterm results. J Thorac Cardiovasc Surg. 2002;123:919-927.
Vascular Health and Risk Management

\section{Publish your work in this journal}

Vascular Health and Risk Management is an international, peerreviewed journal of therapeutics and risk management, focusing on concise rapid reporting of clinical studies on the processes involved in the maintenance of vascular health; the monitoring, prevention and treatment of vascular disease and its sequelae; and the involvement of

\section{Dovepress}

metabolic disorders, particularly diabetes. This journal is indexed on PubMed Central and MedLine. The manuscript management system is completely online and includes a very quick and fair peer-review system, which is all easy to use. Visit http://www.dovepress.com/ testimonials.php to read real quotes from published authors. 